Article

\title{
Improvement for Convolutional Neural Networks in Image Classification Using Long Skip Connection
}

\author{
Hong Hai Hoang * and Hoang Hieu Trinh
}

check for updates

Citation: Hoang, H.H.; Trinh, H.H. Improvement for Convolutional Neural Networks in Image Classification Using Long Skip Connection. Appl. Sci. 2021, 11, 2092. https://doi.org/10.3390/app 11052092

Academic Editor: Manuel Armada

Received: 1 February 2021

Accepted: 22 February 2021

Published: 26 February 2021

Publisher's Note: MDPI stays neutral with regard to jurisdictional claims in published maps and institutional affiliations.

Copyright: (c) 2021 by the authors. Licensee MDPI, Basel, Switzerland. This article is an open access article distributed under the terms and conditions of the Creative Commons Attribution (CC BY) license (https:/ / creativecommons.org/licenses/by/ $4.0 /)$.
School of Mechanical Engineering, Hanoi University of Science and Technology, Hanoi 100000, Vietnam; hieudaic@gmail.com

* Correspondence: hai.hoanghong@hust.edu.vn; Tel.: +84-093-449-3466

\begin{abstract}
In this paper, we examine and research the effect of long skip connection on convolutional neural networks (CNNs) for the tasks of image (surface defect) classification. The standard popular models only apply short skip connection inside blocks (layers with the same size). We apply the long version of residual connection on several proposed models, which aims to reuse the lost spatial knowledge from the layers close to input. For some models, Depthwise Separable Convolution is used rather than traditional convolution in order to reduce both count of parameters and floatingpoint operations per second (FLOPs). Comparative experiments of the newly upgraded models and some popular models have been carried out on different datasets including Bamboo strips datasets and a reduced version of ImageNet. The modified version of DenseNet 121 (we call MDenseNet 121) achieves higher validation accuracy while it has about $75 \%$ of weights and FLOPs in comparison to the original DenseNet 121.
\end{abstract}

Keywords: convolutional neural network; long skip connection; depthwise separable convolution

\section{Introduction}

Wood in general and bamboo in particular have become one of the most popular materials today due to their environmental friendliness. Because of its popularity, product quality requirement becomes more important and can be a crucial aspect in the industrial production line. Many studies in the field of defect detection and classification, in regard of digital image processing, aim to minimize or replace human vision and decision methodologies with artificial techniques [1]. Recent image processing-based methods could deal with defect classification at a decent performance level but they were limited to detecting simple, distinctive defects from the background. Silvén, O [2] used self-organizing map (SOM) for discriminating between sound wood and defects. Qi, X. [3] proposed an algorithm by combination with Blob analysis algorithm and image preprocessing approach to detect the defects. Haindl, M. [4] used fast multispectral texture defect detection method based on the underlying three-dimensional spatial probabilistic image model. Xiansheng [5] provided an online method of bamboo defect inspection and classification. Wang, X. [6] proposed a new surface grading approach by integrating the color and texture of bamboo strips based on Gaussian multi-scale space.

Recent studies in the deep learning field have proved that most problems in machine vision that were difficult in the traditional computer vision methods, were solved using convolutional neural network (CNNs). Krizhevsky et al. [7] first introduced CNN with AlexNet that trained with the difficult ImageNet dataset, solved the classification task with top- 1 and top- 5 error rates of $37.5 \%$ and $17.0 \%$, which was by far better than previous works. Simonyan and Zisserman [8] proved that CNNs had to have a deep network of layers in order for this hierarchical representation of visual data to work. However, stacking multiple convolution layers can cause the problem of vanishing gradient, making the model difficult to learn. ResNet [9] and Highway Networks [10] introduced the idea that vanishing gradient problem could be tackled by by-passing the signal from layer to layer 
by connections. Deeper architectures were created using skip connections and achieved better accuracy. DenseNet [11] utilized these connections by creating short paths between every layer in its architecture. Skipping effectively simplifies the network, using fewer layers in the initial training stages. This accelerates learning by reducing the impact of vanishing gradients, as there are fewer layers to propagate through. The network then gradually restores the skipped layers as it learns the feature space. Towards the end of training, when all layers are expanded, it stays closer to the manifold and thus learns faster. Xception [12] and MobileNet v1 [13] presented Separable Convolutional layer, which significantly reduced computational cost, comparing to traditional Convolutional layer, with only a small decrease in accuracy.

In this paper, we study effect of long skip connection in several convolution neural networks while performing image classification tasks. The models with the long connection are proved to be more robust to converge, have better performance than its counterpart, and can be applied to every model. For DenseNet and ResNet, we change traditional convolution by Depthwise Separable Convolution for the purpose of reducing parameters and floating-point operations per second (FLOPs). We observe the long skip connections accelerate the convergence of the learning process, while performing slightly better in comparison with tested models.

This paper is organized in the following context: Section 2 shows the works related to our network. Our modified network is shown in Section 3. Section 4 presents the experimental results and analysis. The discussion, conclusions and further work are detailed in the last two sections.

\section{Related Works}

Skip connection has become a standard in today's convolutional architectures. Short skip connections are used along with consecutive convolutional layers that do not change the dimension inside blocks of layers until a convolution with stride equal to two or pooling layers; while long skip connections usually exist in the architectures that are symmetrical.

Highway networks used learned gating mechanisms (as a first form of skip connection) to regulate information flow, inspired by Long Short-Term Memory (LSTM) [14] recurrent neural networks. The gating mechanisms allowed neural networks to have paths for information to follow across different layers ("information highways"), using and spreading knowledge from previous layers. The idea was explained again in ResNet that: the deeper layers are identity mapping, and the other layers are learned from the shallower layers. By this idea, ResNet's authors created a deep residual learning architecture with 152 layers and won ILSVRC 2015 with an incredible error rate of 3.6\%. Another popular model that utilized the effect of skip connection is DenseNet. This architecture heavily used feature concatenation so as to ensure maximum information flow between layers in the network. This was achieved by connecting via concatenation of all layers directly with each other, as opposed to ResNet. The DenseNet's authors claimed that their idea led to an enormous amount of feature channels on the last layers of the network, to more compact models, and extreme feature reusability.

U-Net [15] performed long skip connections between layers in encoder and decoder; while the encoder did the convolution work, the decoder did the deconvolution (transpose convolution), which is opposed to the first path. By introducing skip connections in the encoder-decoder architecture, fine-grained details can be recovered in the prediction. Even though there was no theoretical justification, symmetrical long skip connections worked incredibly effectively in dense prediction tasks (medical image segmentation).

For the task of improving a model's computational performance, Depthwise Separable Convolution was first introduced in Xception as an Inception [16] module and placed throughout the deep learning architecture. Depthwise Separable Convolution is made by a Depthwise Convolution (channel-wise $D_{K} \times D_{K}$ spatial convolution) followed by a pointwise convolution $\left(1 \times 1\right.$ convolution) to change the dimension. With $D_{K} \times D_{K}$ equals $3 \times 3$, Depthwise Separable Convolution lowers computation effort, with only a 
small reduction in accuracy. MobileNet adopts the idea and built network architecture that even outperforms SqueezeNet [17] and AlexNet while the multi-adds and parameters were much fewer. MobileNet v2 [18] introduced two new features to the base architecture of the first version, which were linear bottlenecks between the layers, and shortcut connections between the bottlenecks. The latest MobileNet (v3) [19] family member used h-swish instead of sigmoid function [20] and mobile-friendly Squeeze-and-Excitation blocks [21], together with model architecture auto searching. The two later versions of MobileNet family achieved about 75\% accuracy and less than 5 ms latency (running on Pixel4 Edge TPU mobile).

Recent model architectures have focused on balancing accuracy and latency; MnasNet [22] was the first to introduce automated neural architecture search approach for designing mobile models using reinforcement learning. EfficientNet [23] uniformly scaled each dimension (width, depth and resolution) with a fixed set of scaling coefficients, achieving much better accuracy and efficiency than previous CNNs.

\section{Methodology}

Consider $x_{0}$ as input image, passed through a convolutional network, $L$ is number of layers in the network, while $H_{l}$ is the non-linear transformation of $l^{\text {th }}$ layer. Output of $l^{\text {th }}$ is $x_{l}$.

\subsection{Skip Connection}

Traditional feed-forward convolutional networks connect the output of the $l^{\text {th }}$ layer as input to the $(l+1)^{\text {th }}$ layer, which gives rise to the following layer transition: $x_{l}=H_{l}\left(x_{l-1}\right)$. The ResNet's authors added a skip connection that bypasses the non-linear transformations with a function as identity as shown in Figure 1:

$$
x_{l}=H_{l}\left(x_{l-1}\right)+x_{l-1}
$$

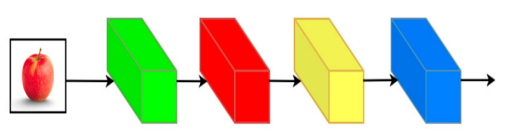

(a) Traditional convolution

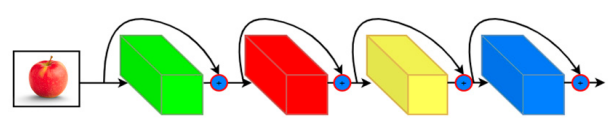

(b) Residual connection, using element-wise addition

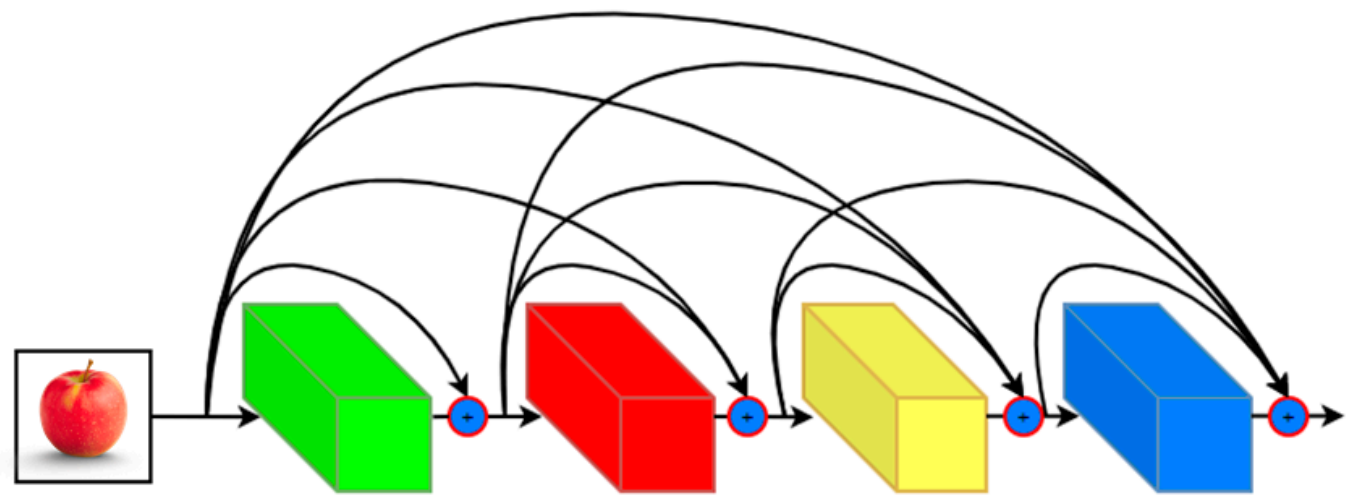

(c) Dense connections, using concatenate

Figure 1. Simple representation of types of convolutions from layer to layer, note that layers have the same height and width.

An advantage of ResNet was that the gradient can flow directly through the identity function from early, close to input layers to the subsequent layers. 


\subsection{Dense Connections}

Huang et al. [11] introduced dense connections that connected every layer in feedforward direction. Different from ResNet, DenseNet did not sum the output feature maps of the layer with the incoming feature maps but concatenated them instead. Consequently, the equation for the $l^{\text {th }}$ layer:

$$
x_{l}=H_{l}\left(\left[x_{0}, x_{1}, \ldots, x_{l-1}\right]\right)
$$

where $\left(\left[x_{0}, x_{1}, \ldots, x_{l-1}\right]\right)$ is the concatenation of output feature map of layers $0,1, \ldots, l-1$.

Since this grouping of feature maps cannot be done when the sizes of them are different, DenseNet is divided into Dense Blocks, where the dimensions of the feature maps remain constant within a block, but the number of filters changes between them. Huang et al. [11] also created Transition Layers, which stay between blocks and take care of the downsampling by applying a Batch Normalization (BN), a $1 \times 1$ convolution and a $2 \times 2$ pooling layers.

Another important feature of DenseNet is the growth rate. As defined in [11], the $l^{\text {th }}$ layer had $k_{0}+k \times(l-1)$ input feature map; where $k_{0}$ was the number of feature maps at input layer, and $k$ was the growth rate.

\subsection{Depthwise Separable Convolution}

Srivastava et al. [10] and Huang et al. [11] proposed Separable Convolutions layer ("s-conv" for short), which performed first a Depthwise Spatial Convolution (which acts on each input channel separately) followed by a pointwise convolution which mixes together the resulting output channels.

A standard 2D convolutional layer takes $D_{H} \times D_{W} \times M$ as input feature map I and produces a $D_{h} \times D_{w} \times N$ output feature map $O$ where $D_{W}$ and $D_{H}$ are the spatial width and height of a input feature map, $M$ is the number of input depth, $D_{w}$ and $D_{h}$ are the spatial width and height of a output feature map and $N$ is the number of output depth.

Kernel $K$ performs convolution on input feature map (with zero padding and stride one), has size $D_{K} \times D_{K} \times M \times N$, where $D_{K}$ is the spatial dimension of the kernel (assumed to be square) and $M$ is the number of input channels and $N$ is the number of output channels as defined. Normal convolutions have the computational cost of:

$$
L_{\text {conv2d }}=D_{K} \times D_{K} \times M \times N \times D_{h} \times D_{w}
$$

The computational cost depends multiplicatively on the number of input channels $M$, the number of output channels $N$, the kernel size $D_{K} \times D_{K}$ and the feature map size $D_{h} \times D_{w}$.

Depthwise Separable Convolution splits a kernel into 2 separate kernels that do two convolutions: the Depthwise Spatial Convolution and the pointwise convolution. Depthwise Spatial Convolution is the channel-wise $D_{K} \times D_{K}$ spatial convolution. Pointwise convolution is the $1 \times 1$ convolution to change the dimension. Separable convolutions have the computational cost:

$$
L_{\text {separable conv2d }}=D_{K} \times D_{K} \times M \times D_{h} \times D_{w}+M \times N \times D_{h} \times D_{w}
$$

Performing division, the computational reduction is:

$$
\frac{\mathrm{L}_{\text {separable conv2d }}}{\mathrm{L}_{\text {conv } 2 d}}=\frac{1}{N}+\frac{1}{D_{K}^{2}}
$$

\subsection{Formatting of Mathematical Components}

DenseNet [11], U-Net [15] and V-Net [24] have showed that convolutional networks can be significantly deeper, more accurate, and simple to train if they contain shortcut connections between layers close to the input and ones close to the output. 
Inspired by these ideas, we first create bottlenecked block with skip connection, dense connectivity and growth rate equaled to 32 similar to DenseNet's architecture and connect every block to eaxh other in feed-forward fashion. We add longer skip connections to pass features from upper layers (blocks) to lower layers (blocks). Generally, the $t^{\text {th }}$ block receives the feature maps of all early blocks $n_{0}, n_{1}, \ldots, n_{t-1}$. The function of the $t^{\text {th }}$ layer is presented as follows:

$$
n_{t}=H_{l, n_{t}}\left(x_{l, n_{t-1}}\right)+\sum_{i=0}^{t-1} n_{i}
$$

where $(t-1)^{\text {th }}$ block has 1 layer.

We use $3 \times 3$ separable convolution layer instead of $3 \times 3$ normal convolution, as it reduces number of parameters as well as FLOPs, thus lowering computational effort.o

\subsection{Bottlenecked Layers}

It has been shown in $[9,16]$ that a $1 \times 1$ convolution can be presented as bottleneck layer before each $3 \times 3$ convolution to reduce the number of input feature maps, therefore improving computational performance. We design our building blocks as this method, stacking $1 \times 1$ conv, $3 \times 3$ separable-conv, then $1 \times 1$ conv, where $1 \times 1$ conv(s) function to reducing and restore dimensions, while $3 \times 3 \mathrm{~s}$-conv(s) do the convolution with lower input/output dimensions.

\subsection{Upstream and Pooling Layers}

An important aim of convolution networks is to downsample layers in which feature maps sizes change. To deal with the blocks' depth difference, we add $1 \times 1$ conv to match block to block depth. Upstream2D and MaxPooling2D layers are added on the connections between blocks as shown in Figure 2.



Figure 2. Connection between 2 blocks with upstream and pooling layers (called Add block). BN denotes Batch Normalization and the normal line represents long skip connection between blocks while dash line states that there could be additional block(s) between block F and K.

\subsection{M-DenseNet Architecture}

Figure 3 illustrates the simple M-DenseNet architecture. Similar to DenseNet, we create dense connection between layers in each block, then add transition layer(s) to perform downsampling. However, we change all $3 \times 3$ conv layers in DenseNet to s-conv layer, then add Add block as shown in Table 1. 


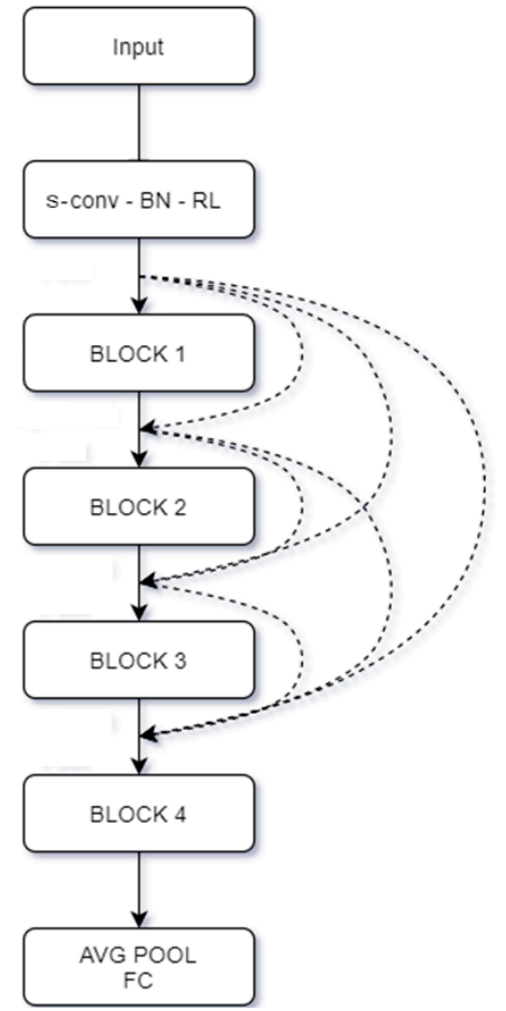

(a) Proposed model

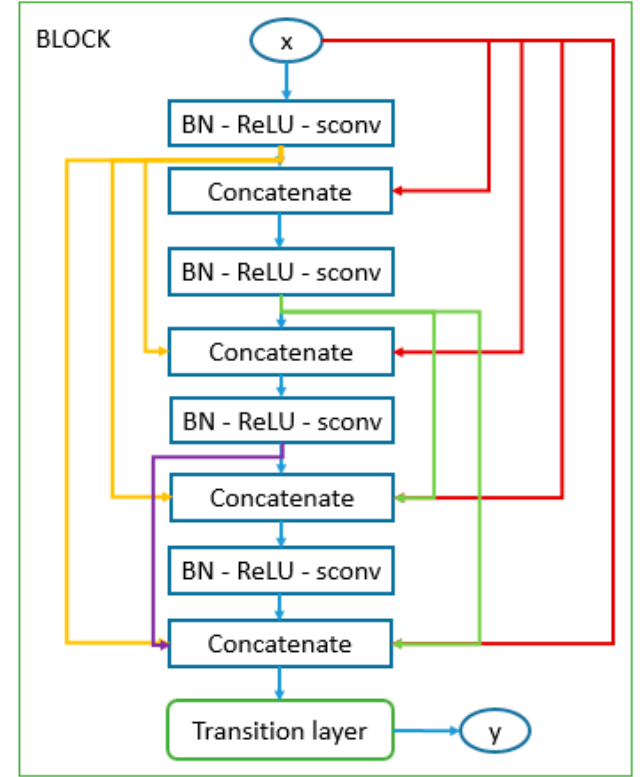

(b) DenseNet block

Figure 3. An overview of model architecture. Note that the skip connections are created between blocks (layers with different shapes). Our model can be considered as a larger version of connection of DenseNet with separable convolution instead of normal 2D convolution.

Table 1. MDenseNet's architectures.

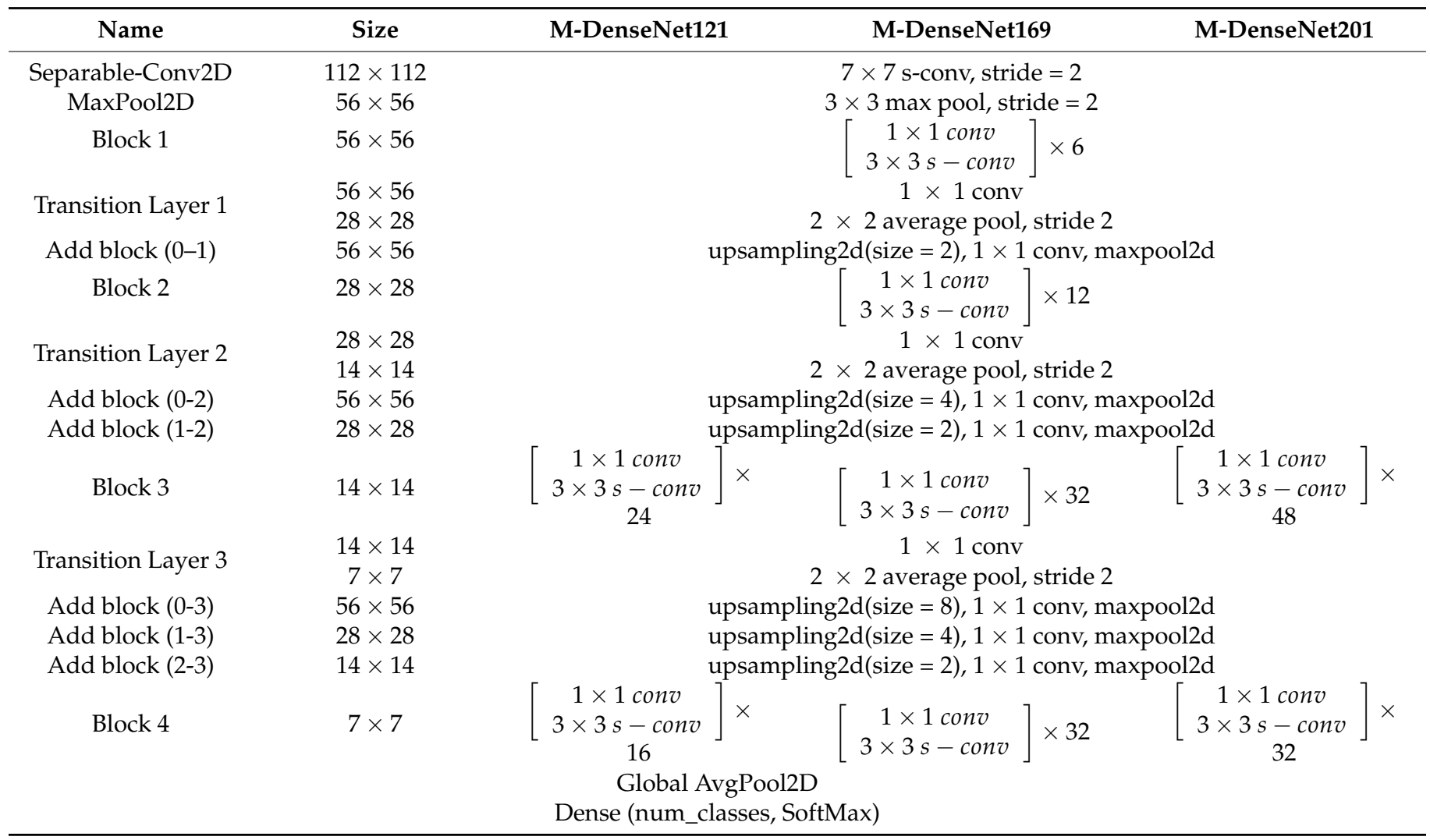


Table 1 shows different configuration of the M-DenseNet. Blocks are shown in bracket with number of repetitions. Upsampling $2 d$ with $1 \times 1$ conv $2 d$ helps add blocks match dimension. Introduced initially before the Block 1 , a convolution with 64 output channels, $7 \times 7$ kernel size and 2 strides are applied on the input image. The block architecture, as shown in Figure 2, follows the idea of Deeper Bottleneck Architectures [11]. Add block $(m-n)$ means that we create long connection between block $m$ and block $n$, block 0 is the output feature map of the first MaxPooling2D layer and block $n$ is the output feature map of Transition Layer $\mathrm{n}^{\text {th }}$.

\section{Experiments and Results Analysis}

\subsection{Case Study on the Bamboo Strips Dataset}

\subsubsection{Build Dataset}

The Bamboo images are taken by using high speed Area Color camera (BASLERacA1920-150uc, 200 frames per second) with a lens of $8 \mathrm{~mm}$ focal length (TAMRON), a frame grabber and a PC. The camera is fixed above the bamboo strip and set focus on the surface. Because of the importance of keeping light in an undisturbed environment, a square shaped LED light is fixed above the bamboo strips. The type of lights, with the addition of a black box as shown in Figure 4 below, are effective against reflection and shadow, as well as the disturbing light from the environment.

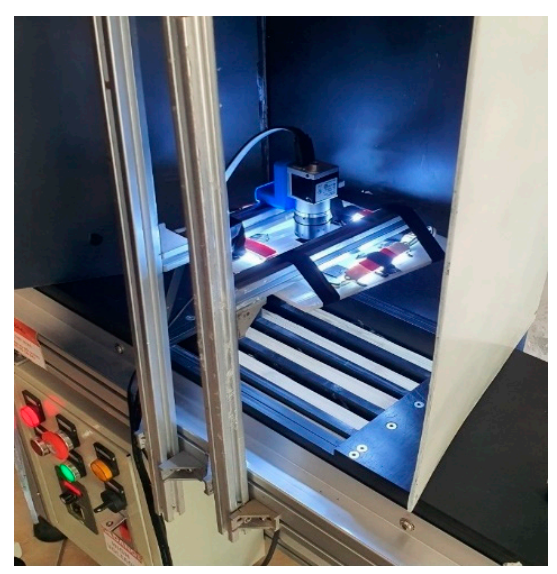

Figure 4. Camera and light system.

Typical bamboo strips have $2 \mathrm{~cm}$ width, so in order to improve production speed, we recorded images of five parallel strips over the conveyor. To deal with lens distortion, we calibrated the camera and obtain its matrix together with distortion coefficients, by the help of OpenCV library [25]. The images are then split into five equals parts (removing unwanted areas), containing each bamboo strip with similar height and width, as described in Figure 5.

We build a Bamboo strips dataset (about 25,000 images), which contains seven classes that are classified manually. The dataset can be accessed at https://github.com/hieuth133 /Bamboo, accessed on 24 January 2021. The images shape is $260 \times 500 \times 3$ and the dataset contains seven classes: one for good bamboo strips, five classes contain non-qualified bamboo strips images (detailed in Section 3.1), and the last one is background (images of conveyor). Example of bamboo defect images are shown in Figure 6. Images are cropped to $224 \times 224 \times 3$ with per-pixel mean subtracted. Because the number of images containing defect is minimal compared to the good bamboo strips images, we upsample the defect bamboo classes by using image augmentation. Using Keras ImageDataGenerator library [26], we generate new images by horizontal and vertical flipping, rotating ( \pm 2 degree), shifting height and width, changing brightness. Both original and generated images are used together to form the Bamboo dataset. Figure 7 points out the distribution of number of images from seven classes in percentages. 

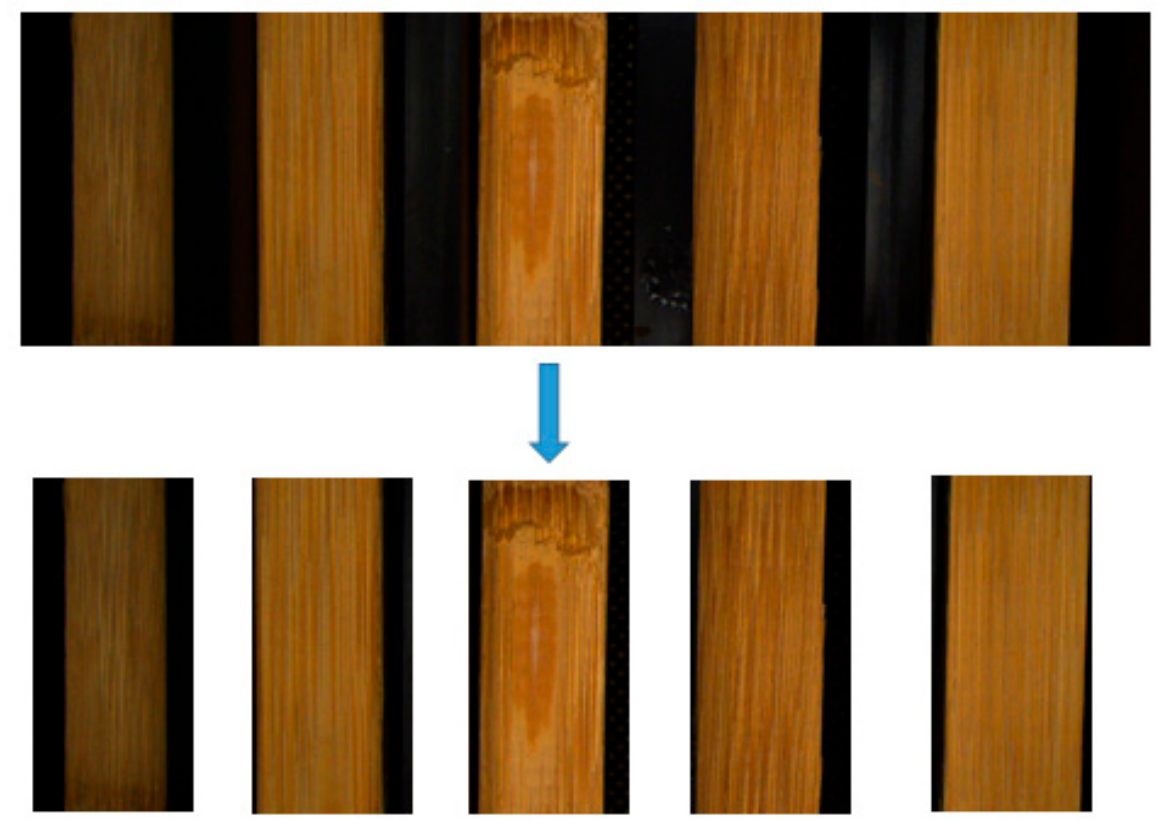

Figure 5. Initial image to 5 divided images (from top to bottom).

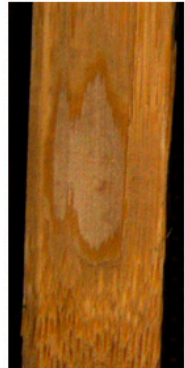

(a)

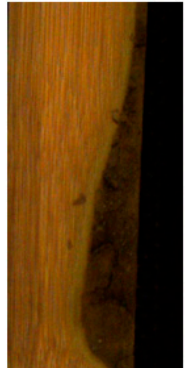

(b)

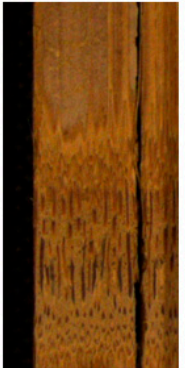

(c)

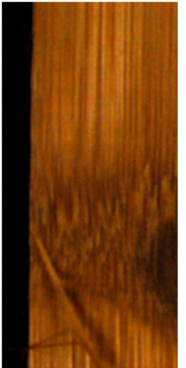

(d)

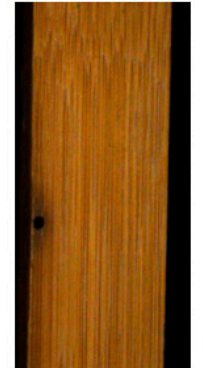

(e)

Figure 6. Example of non-qualified bamboo strips class images, from left to right: (a) stick with inner side, (b) stick with outer skin, (c) cracked, (d) defective cut, (e) worm eaten.

\section{Dataset Structure}

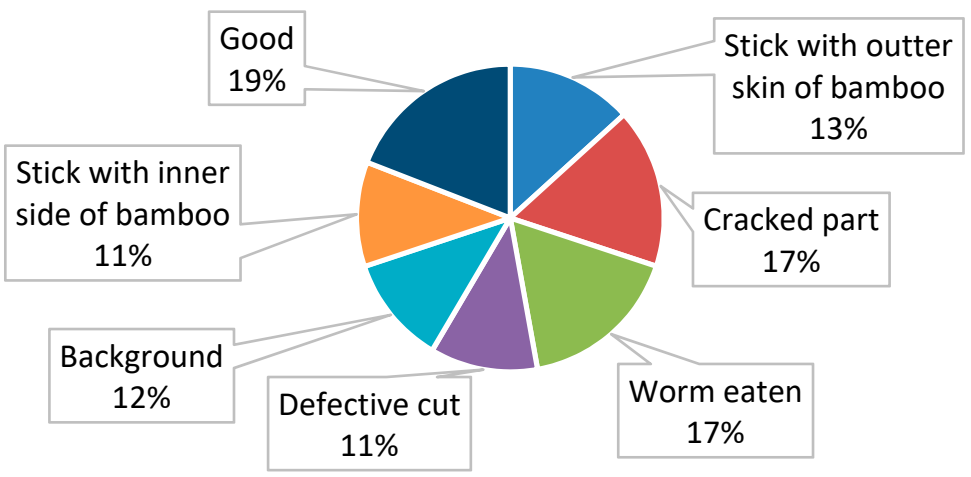

- Stick with outter skin of bamboo

- Cracked part

- Worm eaten

- Defective cut

- Background

n Stick with inner side of bamboo

- Good

Figure 7. Bamboo dataset structure. 


\subsubsection{Training Model}

We evaluate the M-DenseNet on our Bamboo strips dataset, as shown in Figure 8.

We choose batch size of 32 for 30 epochs and stochastic gradient descent (SGD), with learning rate equal to $10^{-3}$, divided by 10 at epoch 10 and 20, weight decay $=10^{-6}$, momentum $=0.9$, and Nesterov momentum is applied. Training time is about 3 hours on Tesla V100-SXM2 with 16GB of VRAM, and computation capability is 7.0. Table 2 shows the results of M-DenseNet(s), DenseNet(s) and ResNet(s) trained on Bamboo dataset. All models' accuracies are qualified for bamboo industry ( $\geq 95 \%)$, and M-DenseNet utilizes parameters more efficient than other models, which appears in Figure 9 and image prediction as shown in Figure 10. Model accuracy is defined as an average number of items correctly identified as either truly positive or truly negative out of the total number of items:

$$
\frac{\sum_{i=1}^{k} \frac{t p_{i}+t n_{i}}{t p_{i}+t n_{i}+f p_{i}+f n_{i}}}{k}
$$

where $k$ is number of classes, " $t$ " is true, " $f$ " is false, " $p$ " is positive and " $n$ " is negative.

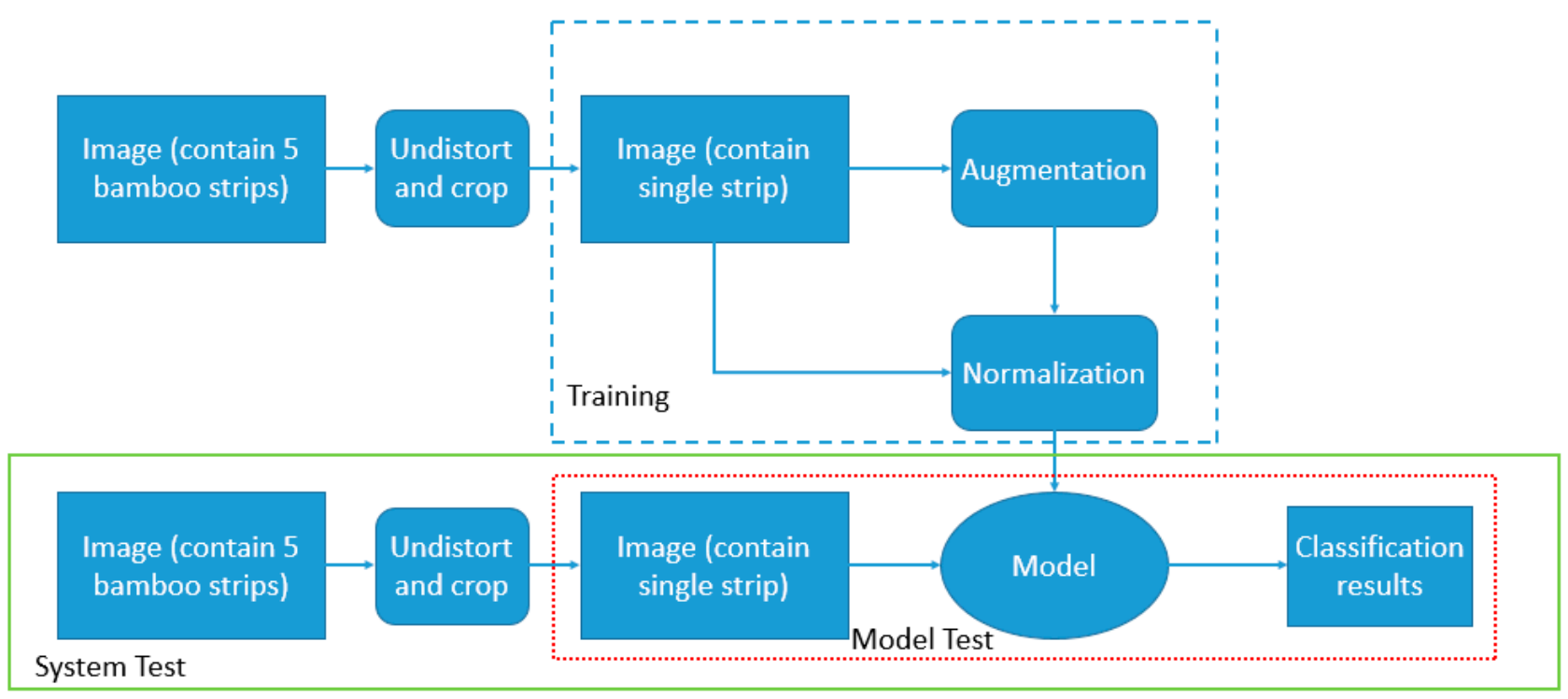

Figure 8. Training and testing method for Bamboo image classification.

Table 2. Accuracy and floating-point operations per second (FLOPs) achieve by M-DenseNet in comparison with the other models.

\begin{tabular}{cccc}
\hline Model & $\begin{array}{c}\text { Parameter } \\
\left(10^{6}\right)\end{array}$ & $\begin{array}{c}\text { FLOPs } \\
\left(10^{6}\right)\end{array}$ & $\begin{array}{c}\text { Accuracy on } \\
\text { Validation Set } \\
(\mathbf{\%})\end{array}$ \\
\hline DenseNet121 & 7.05 & 14.97 & 96.73 \\
DenseNet169 & 12.66 & 27.76 & 98.16 \\
DenseNet201 & 18.34 & 39.90 & 97.83 \\
ResNet50 & 23.50 & 47.00 & 96.37 \\
ResNet101 & 42.00 & 85.00 & 96.58 \\
MDenseNet121 & 5.51 & $\mathbf{1 0 . 8 3}$ & 99.46 \\
MDenseNett169 & 10.42 & 20.50 & 99.46 \\
MDenseNet201 & 15.71 & 30.94 & $\mathbf{9 9 . 4 9}$ \\
\hline
\end{tabular}

Numbers in bold represent best result. 

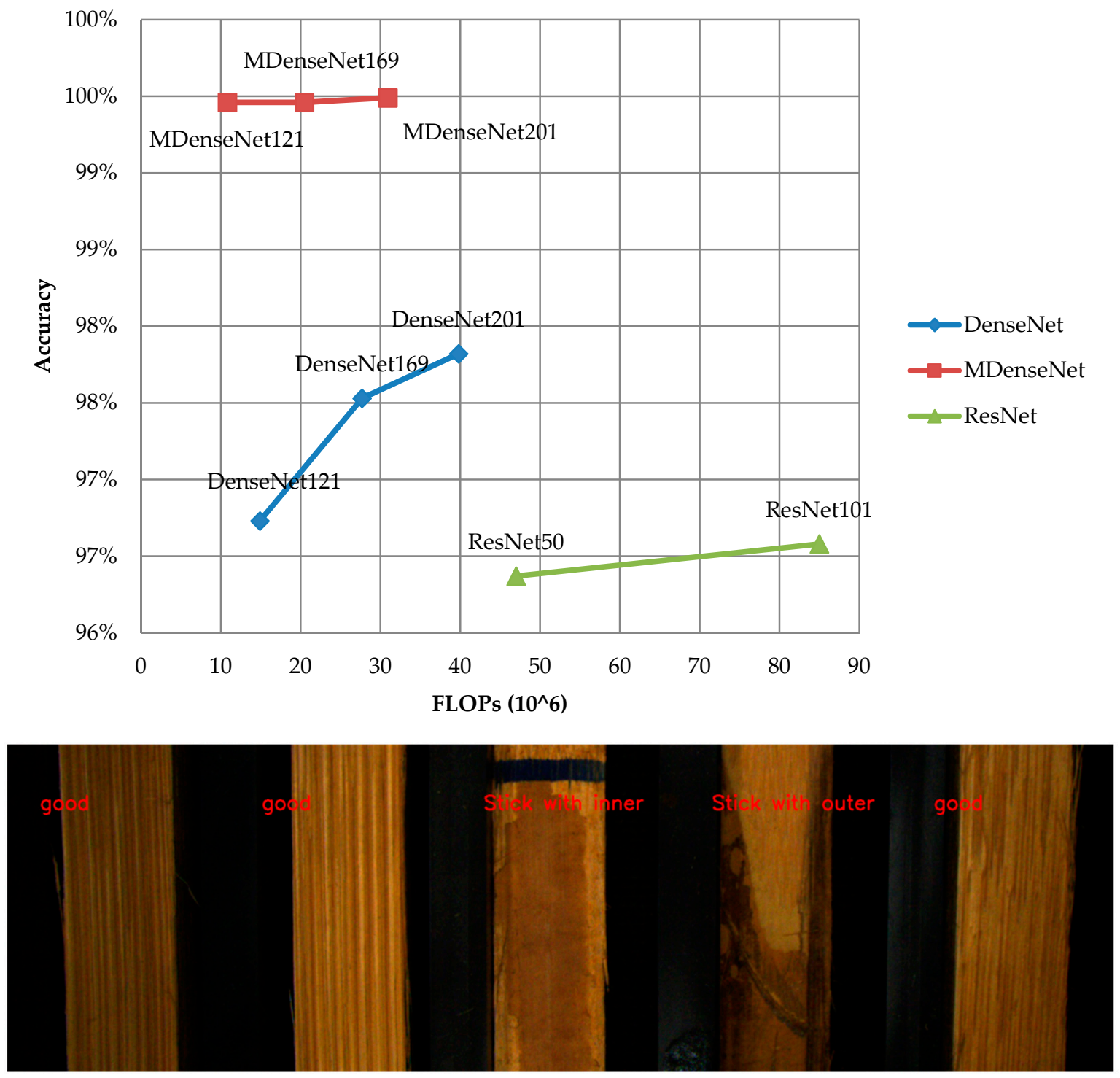

Figure 10. Image prediction final result.

\subsection{Case Study on the Reduced Version of ImageNet}

We also test the M-DenseNet and DenseNet families without/with long skip connection and s-conv. We use another dataset, which is a reduced version of ImageNet [27] with 100 classes and about 500 images in each class. The dataset is also accessible via the same github link above (Section 4.1.1). All models are trained by the same technique with extra augmentation [28-30] and normalization [31]; batch size is 32, Adam optimizer, 60 epochs and learning rate is $10^{-3}$.

Table 3 presents the value of accuracy and FLOPs in comparison between DenseNet and M-DenseNet. By observation, models with long skip connection converge faster, as shown in Figure 11 and has a better overall result. Parameters and FLOPs of M-DenseNet family equal roughly $80 \%$ of original DenseNet, while the accuracy increases about 3-5\%. 
Table 3. Accuracy and FLOPs achieved by M-DenseNet in comparison with DenseNet.

\begin{tabular}{cccc}
\hline Model & $\begin{array}{c}\text { Parameter } \\
\left(10^{6}\right)\end{array}$ & $\begin{array}{c}\text { FLOPS } \\
\left(10^{6}\right)\end{array}$ & $\begin{array}{c}\text { Accuracy on } \\
\text { Validation set } \\
(\%)\end{array}$ \\
\hline DenseNet121 & 7.14 & 14.11 & 73.72 \\
DenseNet169 & 12.80 & 25.30 & 74.54 \\
DenseNet201 & 18.51 & 36.57 & 75.65 \\
M-DenseNet121 & $\mathbf{5 . 5 0}$ & $\mathbf{1 0 . 8 3}$ & 78.56 \\
M-DenseNet169 & 10.41 & 20.50 & 78.86 \\
M-DenseNet201 & 15.71 & 30.94 & $\mathbf{7 9 . 4 2}$ \\
\hline
\end{tabular}

Numbers in bold represent best result.

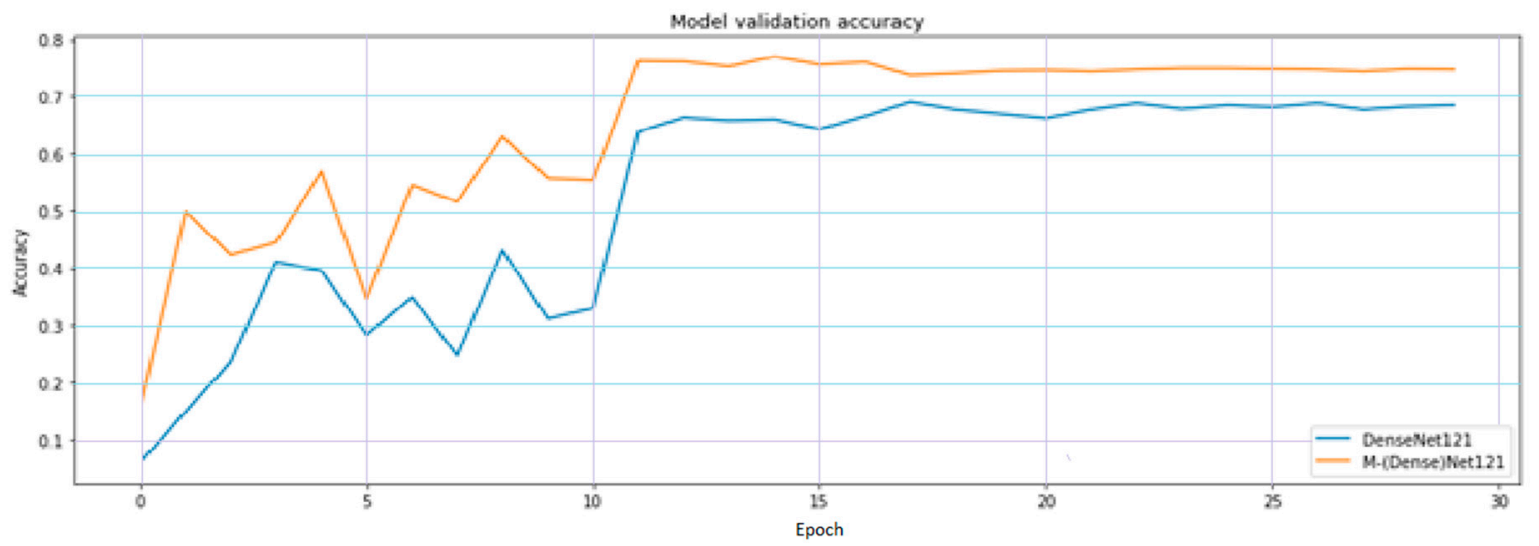

Figure 11. Comparison between DenseNet121 and M-DenseNet121 in terms of model validation accuracy from epoch 0 to 30.

Testing usage with other models: We make modifications to other models, similar to what we have done to create MDenseNet in Section 3.7. The models are EfficientNet [23] and MobileNet v1, v2, v3 [13,18,19]. We use a reduced version of ImageNet and training technique the same as that explained in Section 4.2. Below is the detail of comparison.

Figures 12 and 13 point out that our modifying models perform better than the original ones with small decrease in FLOPs (6-3\% for MobileNet and 2-1\% for EfficientNet). This proves the effectiveness of the long skip connections to performance of CNN.

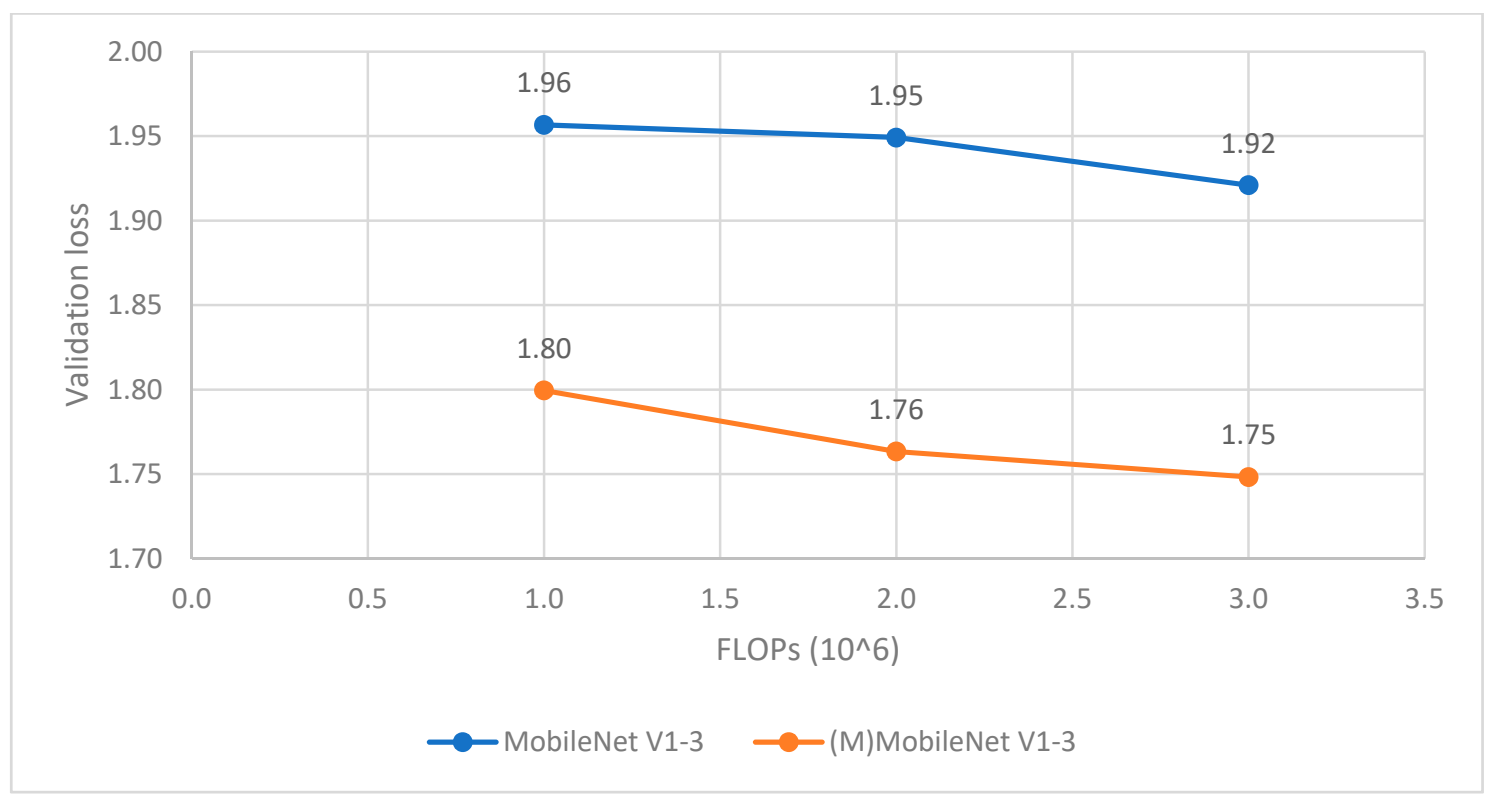

Figure 12. Comparison between MobileNet and (M) MobileNet (V1-V3, right to left, respectively). 


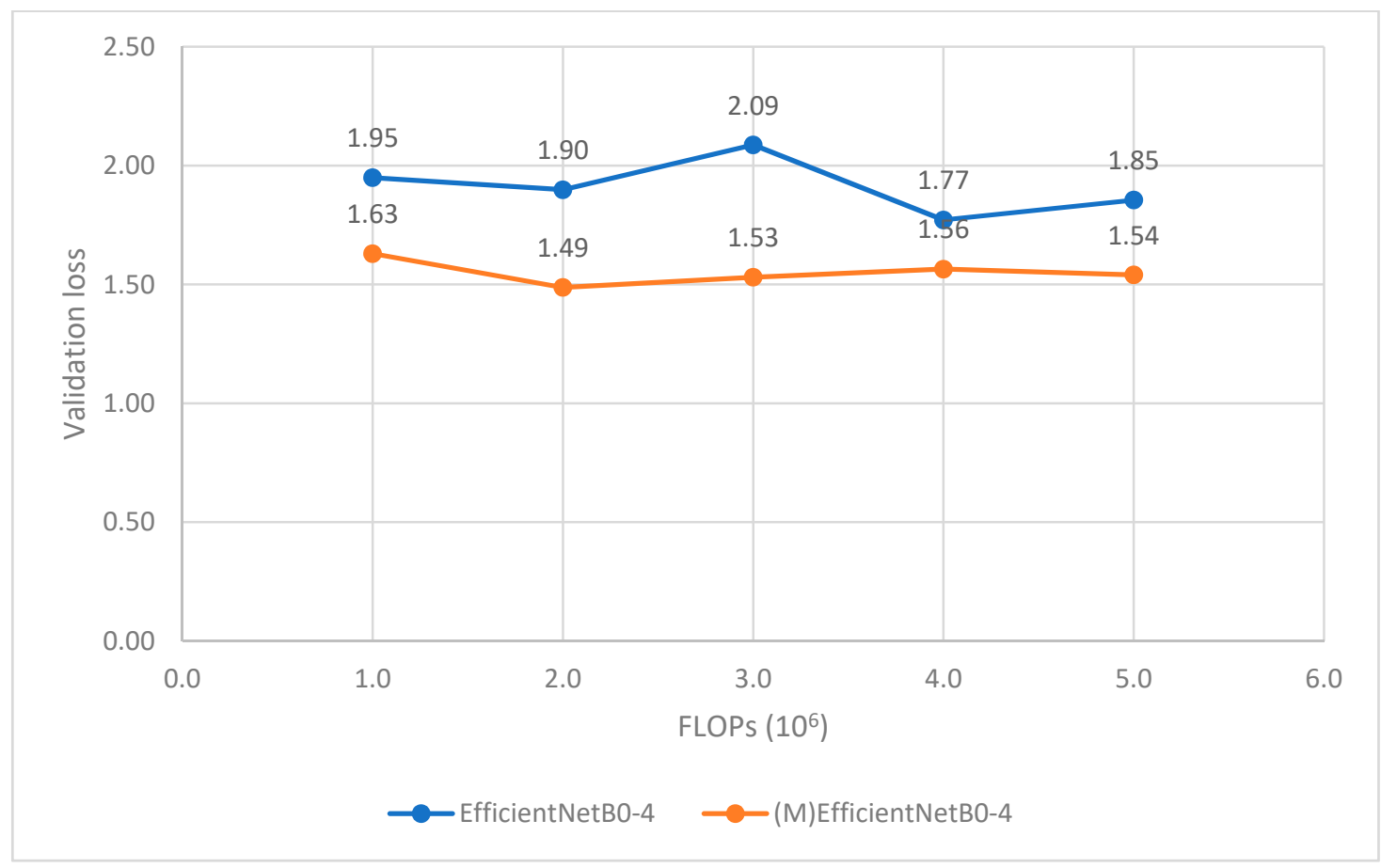

Figure 13. Comparison between EfficientNet and (M) EfficientNet (B0-B4, left to right, respectively).

\subsection{Ablation Study}

We conducted an ablation study to point out the effectiveness of long skip connection to the performance of the models. We used EfficientNet B0 and three modified models based on EfficientNet B0 for testing.

The configuration for training and dataset is the same as that explained in Section 4.2. Figure 14 shows the validation loss while training 4 models in 60 epochs. Models with more long skip connections perform better than ones with less long connections.

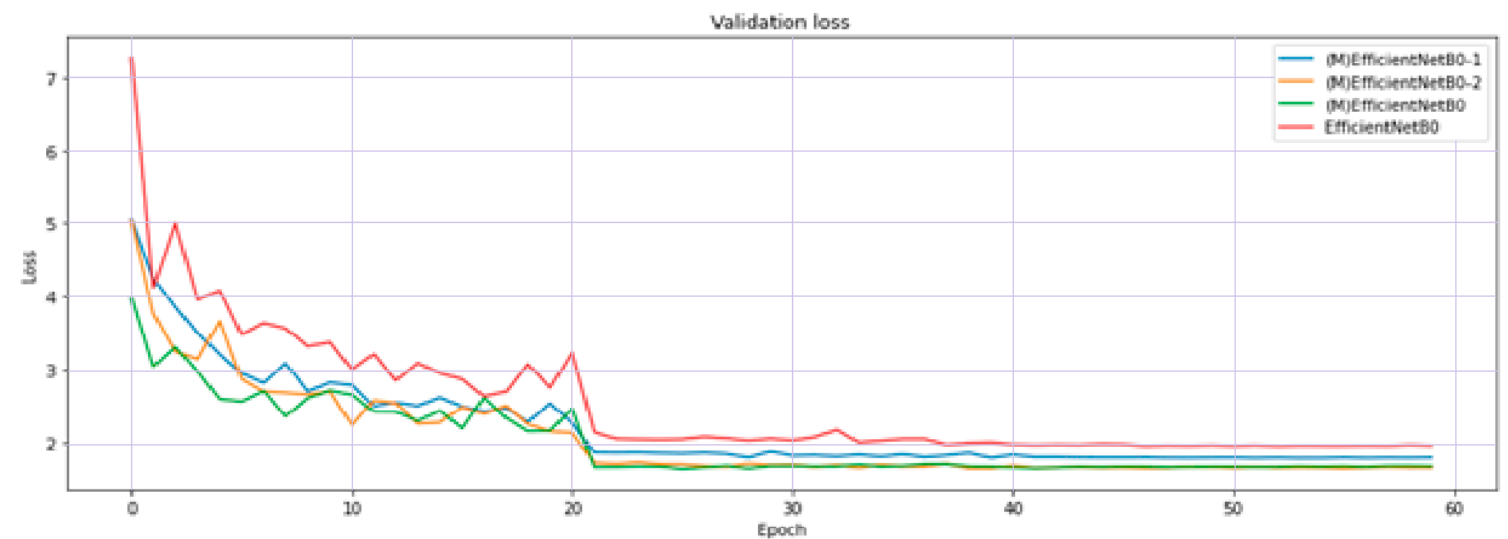

Figure 14. Validation loss comparison between EfficientNet B0 and our modification based on EfficientNet B0 ( 3 models); EfficientNet B0 is the base model; (M) EfficientNet B0 is our modification model, add blocks are added that connect all blocks similar to DenseNet (Figure 3.b); (M) EfficientNet B0-1 has only connections between consecutive blocks (Block N Block N+1); (M) EfficientNet B0-2 has connections between consecutive blocks and blocks separated by 1 block (Block N Block N+1; Block N - Block N+2). 


\section{Discussion}

As noted above, the modification adds to the model and turns it into a larger version of DenseNet. The upgrade seems to be small but it led to notable consequences. We present a few discussions and experiments to prove the efficiency of our proposed method.

Feature reuse: Generally, the feature maps close to the input detect small or finegrained detail, whereas feature maps close to the output of the model capture more general features. The connection between blocks encourages layers to learn both small detail and general nature of the object. The ablation study also proves the effect of skip connections that is more connections results in better performance.

Adaptability: The method of adding long skip connections has the ability to fit well to other models, from lightweight one like MobileNet family to well-designed EfficientNet family. Comparative experiments show that the modified models converge faster and have better performance.

Difficulty: In order to perform long skip connection between layers having different size, we have to equal the shape of two layers (detail in Section 3.6). This task can consume large GPU memory capacity and is unable to work with small size GPU, as the shape of feature map of the long skip connection layer is very large.

\section{Conclusions}

In this paper, we have proposed a modification to improve the performance of CNNs by using the effect of long skip connections. It creates long connections between blocks of layers with different sizes of feature map. In our experiment, the models with long skip connections tend to converge faster, without overfitting. The MDenseNet 121 model achieves higher validation accuracy while it has about $75 \%$ of weights and FLOPs in comparison to the original DenseNet 121. Adding long skip connections also helps MobileNets and EfficientNets families to improve their performances (tested with a reduced version of ImageNet).

The models with long skip connections have benefits in comparison to their counterparts, as it enhances feature reuse throughout the models, encourage models to learn both the fine details (coming from layers close to input) and the more general details (coming from layers close to output) of the objects. Moreover, the proposed modification is highly adaptable to many models, from lightweight to heavily parameterized models. As ad limitation, we experience difficulty in training big models, which is a result of the large shape of the feature map in the skip connection.

We will try to control the amount of knowledge when performing long skip connection in further experiments. In future work, we will explore this architecture with deeper layers, while maintaining its performance to apply to different tasks such as segmentation or object detection.

Author Contributions: Supervision: H.H.H.; conceptualization: H.H.T.; methodology: H.H.H. and H.H.T.; validation: H.H.H. and H.H.T.; investigation: H.H.T.; resources: H.H.T. and H.H.H.; writingoriginal draft preparation: H.H.T. and H.H.H.; writing-review and editing: H.H.H. and H.H.T.; project administration: H.H.H.; funding acquisition: H.H.H. All authors have read and agreed to the published version of the manuscript.

Funding: This work was funded by Vietnam Ministry of Education and Training under project number B2020-BKA-02.

Institutional Review Board Statement: Not applicable.

Informed Consent Statement: Not applicable.

Data Availability Statement: MDPI Research Data Policies.

Conflicts of Interest: The authors declare no conflict of interest. 


\section{References}

1. Czimmermann, T.; Ciuti, G.; Milazzo, M.; Chiurazzi, M.; Roccella, S.; Oddo, C.M.; Dario, P. Visual-Based Defect Detection and Classification Approaches for Industrial Applications-A SURVEY. Sensors 2020, 20, 1459. [CrossRef] [PubMed]

2. Silvén, O.; Niskanen, M.; Kauppinen, H. Wood inspection with non-supervised clustering. Mach. Vis. Appl. 2003, 13, 275-285. [CrossRef]

3. Qi, X.; Li, X.; Zhang, H. Research of paper surface defects detection system based on blob algorithm. In Proceedings of the IEEE International Conference on Information and Automation (ICIA), Yinchuan, China, 26-28 August 2013.

4. Haindl, M.; Grim, J.; Mikeš, S. Texture Defect Detection. In Computer Analysis of Images and Patterns; Kropatsch, W.G., Kampel, M., Hanbury, A., Eds.; Springer: Berlin/Heidelberg, Germany, 2007; Volume 4673, pp. 987-994.

5. Xiansheng, Q.; Feng, H.; Qiong, L.; Xin, S. Online defect inspection algorithm of bamboo strip based on computer vision. In Proceedings of the IEEE International Conference on Industrial Technology 2009, Gippsland, Australia, 10-13 February 2009.

6. Wang, X.; Liang, D.; Deng, W. Surface grading of bamboo strips using multi-scale color texture features in Eigenspace. Comput. Electron. Agric. 2010, 73, 91-98. [CrossRef]

7. Krizhevsky, A.; Sutskever, I.; Hinton, G.E. ImageNet Classification with Deep Convolutional Neural Networks. In Proceedings of the 25th International Conference on Neural Information Processing Systems, Lake Tahoe, NV, USA, 3-6 December 2012.

8. Simonyan, K.; Zisserman, A. Very Deep Convolutional Networks for Large-Scale Image Recognition. arXiv 2015, arXiv:1409.1556.

9. He, K.; Zhang, X.; Ren, S.; Sun, J. Deep Residual Learning for Image Recognition. In Proceedings of the IEEE Conference on Computer Vision and Pattern Recognition (CVPR), Las Vegas, NV, USA, 27-30 June 2016.

10. Srivastava, R.K.; Greff, K.; Schmidhuber, J. Highway Networks. In Proceedings of the ICML Deep Learning Workshop, Lille, France, 6-11 July 2015.

11. Huang, G.; Liu, Z.; Maaten, L.V.D.; Weinberger, K.Q. Densely Connected Convolutional Networks. In Proceedings of the IEEE Conference on Computer Vision and Pattern Recognition (CVPR), Honolulu, HI, USA, 21-26 July 2017.

12. Chollet, F. Xception: Deep Learning with Depthwise Separable Convolutions. In Proceedings of the IEEE Conference on Computer Vision and Pattern Recognition (CVPR), Honolulu, HI, USA, 21-26 July 2017.

13. Howard, A.G.; Zhu, M.; Chen, B.; Kalenichenko, D.; Wang, W.; Weyand, T.; Andreetto, M.; Adam, H. MobileNets: Efficient Convolutional Neural Networks for Mobile Vision Applications. arXiv 2017, arXiv:1704.04861.

14. Hochreiter, S.; Schmidhuber, J. Long Short-term Memory. Neural Comput. 1997, 9, 1735-1780. [CrossRef] [PubMed]

15. Ronneberger, O.; Fischer, P.; Brox, T. U-Net: Convolutional Networks for Biomedical Image Segmentation. arXiv 2015, arXiv:1505.04597.

16. Szegedy, C.; Vanhoucke, V.; Ioffe, S.; Shlens, J.; Wojna, Z. Rethinking the Inception Architecture for Computer Vision. arXiv 2015, arXiv:1512.00567.

17. Iandola, F.N.; Han, S.; Moskewicz, M.W.; Ashraf, K.; Dally, W.J.; Keutzer, K. SqueezeNet: AlexNet-level accuracy with 50x fewer parameters and $<0.5 \mathrm{MB}$ model size. arXiv 2016, arXiv:1602.07360.

18. Mark, S.; Andrew, H.; Menglong, Z.; Andrey, Z.; Chen, L.C. MobileNetV2: Inverted Residuals and Linear Bottlenecks. In Proceedings of the IEEE Conference on Computer Vision and Pattern Recognition (CVPR), Salt Lake City, UT, USA, 18-22 June 2018; pp. 4510-4520.

19. Andrew, H.; Mark, S.; Grace, C.; Chen, B.; Mingxing, T.; Weijun, W.; Yukun, Z.; Ruoming, P.; Vijay, V.; Quoc, V.L.; et al. Searching for MobileNetV3. arXiv 2019, arXiv:1905.02244.

20. Chigozie, E.N.; Winifred, I.; Anthony, G.; Stephen, M. Activation Functions: Comparison of Trends in Practice and Research for Deep Learning. arXiv 2018, arXiv:1811.03378v1.

21. Jie, H.; Li, S.; Samuel, A.; Gang, S.; Enhua, W. Squeeze-and-Excitation Networks. arXiv 2019, arXiv:1709.01507v4.

22. Mingxing, T.; Bo, C.; Ruoming, P.; Vijay, V.; Mark, S.; Andrew, H.; Le, Q.V. MnasNet: Platform-Aware Neural Architecture Search for Mobile. arXiv 2019, arXiv:1807.11626v3.

23. Tan, M.; Le, Q.V. EfficientNet: Rethinking Model Scaling for Convolutional Neural Networks. In Proceedings of the International Conference on Machine Learning, Long Beach, CA, USA, 10-15 June 2019.

24. Milletari, F.; Navab, N.; Ahmadi, S.-A. V-Net: Fully Convolutional Neural Networks for Volumetric Medical Image Segmentation. arXiv 2016, arXiv:1606.04797.

25. OpenCV. Available online: https:/ / opencv.org/ (accessed on 13 June 2020).

26. Keras. Available online: https:/ / github.com/keras-team/keras (accessed on 13 June 2020).

27. Deng, J.; Dong, W.; Socher, R.; Li, L.-J.; Li, K.; Fei-Fei, L. ImageNet: A Large-Scale Hierarchical Image Database. In Proceedings of the IEEE Conference on Computer Vision and Pattern Recognition (CVPR), Miami, FL, USA, 22-24 June 2009.

28. Terrance, D.; Graham, W. Improved Regularization of Convolutional Neural Networks with Cutout. arXiv 2017, arXiv:1708.04552v2.

29. Ekin, D.; Barret, Z.; Dandelion, M.; Vijay, V.; Le, Q.V. AutoAugment: Learning Augmentation Policies from Data. arXiv 2019, arXiv:1805.09501v3.

30. Ekin, D.; Barret, Z.; Jonathon, S.; Le, Q.V. RandAugment: Practical automated data augmentation with a reduced search space. arXiv 2019, arXiv:1909.13719v2.

31. Sola, J.; Sevilla, J. Importance of input data normalization for the application of neural networks to complex industrial problems. IEEE Trans. Nucl. Sci. 1997, 44, 1464-1468. [CrossRef] 